\title{
The use of artificial intelligence in interpreting the results of radiological imaging - opportunities and threats
}

\author{
Authors: Gabriela Ręka ${ }^{1}$, Piotr Machowiec ${ }^{1}$, Halina Piecewicz-Szczęsna ${ }^{2}$ (mentor) \\ ${ }^{1}$ Students' Scientific Association of Chair and Department of Epidemiology and Clinical Research \\ Methodology, Medical University of Lublin, Poland \\ ${ }^{2}$ Chair and Department of Epidemiology and Clinical Research Methodology, Medical University of \\ Lublin, Poland
}

DOI: https://doi.org/10.26800/LV-142-supp5-59

\section{Introduction:}

Artificial intelligence (Al) is the system's ability to interpret data, learn from them, and use this knowledge to perform tasks. The development of modern intelligent systems in radiology has enabled us to use them in interpreting RTG, CT, MRI, and USG images.

\section{Aim:}

The study aims to present the current state of knowledge about the usage of Al in radiology.

\section{Materials and methods:}

An analysis of scientific publications available on the PubMed and Google Scholar platform since 2018 was performed using the keywords linked by logical operators: "artificial intelligence" and "radiology". As a result, 7 scientific publications were used after selection by two independent analysts.

\section{Results:}

The benefits of using Al relate to faster diagnosis, minimizing errors, cutting costs, and providing care for patients with limited access to specialists. Recent research suggests the potential use of deep learning algorithms to identify abnormalities in CT scans in case of head trauma or stroke symptoms, with high accuracy. Furthermore, it is confirmed that the algorithm improves aneurysm detection in datasets. Other levels of its possible application include differentiating breast cancer molecular subtypes based on MRI (radiogenomics), distinguishing by neural networks between pneumonia in COVID-19 and other lung conditions in CT, and improving knee MRI imaging interpretation in cooperation with specialists. Nevertheless, among threats unverified diagnosis, problems with data protection, and the vanishing of radiology as a specialty are enumerated.

\section{Conclusion:}

The use of $\mathrm{Al}$ in radiology has both numerous advantages and disadvantages, so there is a high need for further Al development towards reducing potential threats resulting from its use, with the needs of patients as a priority. The analyzed literature showed that Al might be successfully applied for making the diagnosis in the pathology of different organs alone or with co-interpretation with radiologists.

Keywords: artificial intelligence, diagnosis, radiology 